\title{
Hybrid FE/ANN and LPR approach for the inverse identification of material parameters from cutting tests
}

\author{
Ana Muñoz-Sánchez • Isabel M. González-Farias • \\ Xavier Soldani • M. Henar Miguélez
}

\begin{abstract}
Accuracy of numerical models based in finite elements (FE), extensively used for simulation of cutting processes, depends strongly on the identification of proper material parameters. Experimental identification of the constitutive law parameters for simulation of cutting processes involves unsolved problems such as the complex testing techniques or the difficulty to reproduce the stress triaxiality state during cutting. This work proposes a methodology for the inverse identification of the material parameters from cutting test. Two hybrid approaches are compared. One of them based on FE and artificial neural networks (ANN). The other one based on FE and local polynomial regression (LPR). Firstly, a FE model is validated with experimental data. Then, ANN and LPR are trained with FE simulations. Finally, the estimated ANN and LPR models are used for the inverse identification of material parameters. This identification is solved as an optimization problem. The FE/LPR approach shows good performance, outperforming the FE/ANN approach.
\end{abstract}

Keywords Inverse technique - Cutting simulation · FE . ANN $\cdot$ Local polynomial regression

\section{Nomenclature \\ Parameters of constitutive equation \\ $\sigma_{Y} \quad$ Equivalent stress \\ $A \quad$ Yield limit \\ $B \quad$ Strain hardening coefficient}

A. Muñoz-Sánchez · I. M. González-Farias • X. Soldani •

M. H. Miguélez $(\bowtie)$

Department of Mechanical Engineering,

Universidad Carlos III de Madrid,

Av. de la Universidad 30,

28911 Leganés, Madrid, Spain

e-mail: mhmiguel@ing.uc3m.es

$n \quad$ Strain hardening exponent

$C \quad$ Strain rate hardening coefficient

$m$ Thermal softening coefficient

$\varepsilon^{\mathrm{pl}} \quad$ Equivalent plastic strain

$\dot{\varepsilon}^{\mathrm{pl}} \quad$ Equivalent plastic strain rate

$\dot{\varepsilon}_{0} \quad$ Reference strain rate

$\theta \quad$ Temperature

$\theta_{\text {ref }} \quad$ Reference temperature

$\theta_{\text {melt }}$ Melting temperature

\section{Description of the methodology}

$\mu$

$F_{c}$

$F_{t}$

$\sigma_{11}$

$\sigma_{33}$

$A_{(i)}, B_{(i)}, n_{(i)}$

$\widehat{F}_{c(i)}, \widehat{F}_{t(i)}, \widehat{\sigma}_{11(i)}, \widehat{\sigma}_{33(i)}$

RBFN and LPR models

$X=\left(X_{1}, X_{2}, \ldots, X_{p}\right)^{\prime} \quad$ Predictor input variables

$Y \quad$ Response variable

\section{RBFN}

$K(\bullet) \quad$ Univariate kernel function

$\|\bullet\| \quad$ Euclidean distance

$J \quad$ Number of hidden units

$v_{j} \quad$ Output of the $j$-th hidden unit

$C_{j} \quad$ Vector of centres of the $j$-th hidden unit

$T_{j} \quad$ Width of the kernel function

$\widehat{Y} \quad$ Estimated value of response variable

$w_{0 k} w_{j k} \quad$ Weights associated with the output unit $k$ 


\section{LPR}

$\left\{x_{i}, y_{i}\right\}, i=1,2, \ldots, n$

$w_{i}$

$\beta_{j}$

$d$

$h_{j}$

$K_{h}\left(u_{j}\right)$

$K_{\mathrm{H}}$

Models estimation

$M \quad$ Number of iterations

$N \quad$ Number of observations of the dataset

$N_{v} \quad$ Validation observations

$s \quad$ Number of output variables

$y \quad$ Real value

$\widehat{y} \quad$ Estimated value

MARE Mean absolute relative error

$M S E \quad$ Mean square error

$A P E \quad$ Absolute percentage error

MAPE Mean absolute percentage error

\section{Introduction}

Manufacturing processes should ensure not only competitive time and cost production, but also the development of components with high quality, especially in the case of high responsibility applications. Simulation tools are widely used in all steps of product design, not only for structural calculations, also for processing route design, being this step related with final service behaviour of the component. Numerical models based on finite elements (FE) have been extensively used for machining simulation $[1,2]$, however many aspects related with numerical simulations remain unsolved.

The identification of material parameters for cutting simulation, strongly influent in the numerical results, is an objective that should be achieved. Many problems remain unsolved when a representative constitutive law should be stated. Firstly, the inexistence of experimental tests able to reproduce the special conditions involved during cutting. Current characterization tests induce stress states in the specimen quite different from those obtained during machining. Another problem is performing typical tests at high strain rates (for instance using metallic sheets in tensile tests) for the same material treatment and the same format as used in machining tests (as an example, bars in turning operations). The work path suffered in different manufacture process leads to different mechanical properties. Different works in scientific literature show reasonable doubts related with the convenience of applying constitutive equation parameters obtained in traditional test to simulate cutting processes. The best solution could be using machining tests to identify the constitutive equation parameters [3].

Artificial neural networks (ANN) and local polynomial regression (LPR) techniques could help in the identification of constitutive law parameters for specific machining conditions. These nonparametric techniques require no assumptions about the form of the relationship between a set of variables.

Hence, they are particularly useful when little information about the model structure is available.

ANN have been extensively used in a wide variety of applications related with manufacturing processes (see for instance [4-6]).

On the other hand LPR techniques [7] have not been commonly used for the analysis of manufacturing processes. These statistical tool provide similar characteristics and requirements as ANN to threat complex relationships, with little information about the interdependence of the input and output variables, however their use has been limited to other fields.

Despite of the potentiality of ANN and LPR models, the main drawback of these techniques is the requirement of a large amount of data for training process. In our context, it is really difficult to have a large amount of experimental data. The variety of materials that should be involved to reproduce a wide range of variation of constitutive parameters would be extremely high. Furthermore, it is not possible to control the constitutive parameters of the materials tested. Thus, the use of validated FE models is an alternative way to generate numerical data to find the best ANN and LPR models.

The hybrid FE/ANN approach has been used in different analysis of manufacturing processes and other fields. For instance, Umbrello et al. [8] used this combined method to predict machining induced residual stresses during hard turning; and Chamekh et al. [9] proposed a hybrid procedure for the identification of HILL anisotropic parameters based on deep drawing of a cylindrical cup. Fernández-Fdz and Zaera [10] used the combined approach to predict the penetration behaviour of ceramic targets under the impact of different projectiles.

In this work, we propose a hybrid procedure for the inverse identification of material parameters from cutting tests. The aim is obtaining constitutive equation parameters suitable to be implemented in FE codes for accurate simulation of cutting. Two approaches, one based FE/ ANN and the other one based on FE/LPR are compared in order to find the best hybrid procedure. These approaches are based on the following variables: (1) the forces and the friction coefficient at the interface, measured/estimated during cutting tests. (2) The machining induced residual stresses, measured on the machined surface. These variables 
are highly sensible to variations of the material parameters. Residual stresses were selected because of both its dependence on material parameters and due to their relevance as surface integrity indicators [11].

Thus, the methodology proposed in this work relates experimental data obtained from orthogonal cutting tests and subsequent measurements on the machined workpiece, with the values of the parameters of the constitutive equation. This approach could simplify significantly the experimental devices needed for research in this field.

The proposed FE/ANN and FE/LPR approaches are divided in three different stages. In the first stage, a FE model is developed using experimental data. Then, the validated model is used to perform a great number of simulations, changing the input data and obtaining a wide range of cutting forces and level of residual stresses for the different ranges of material parameters covered. In the second stage, both ANN and LPR models are trained by using a large set of numerical results obtained from FE model. The neural network used is the Radial Basis Function Network (RBFN). Finally, the last stage involves the inverse identification procedure. In this stage the objective is the prediction of the constitutive parameters according to specific values of cutting forces and residual stresses obtained from cutting tests. The identification problem is solved as an optimization problem using the estimated RBFN and LPR models from second stage.

The rest of the article is organized as follows. Section 2 describes the steps of the proposed FE/ANN and FE/LPR procedures. Section 3 describes the FE model and its experimental validation. Theoretical concepts related with RBFN and LPR techniques are briefly explained in section fourth. Sections 5 shows the results, and finally section 6 summarizes the conclusions of the work.

\section{Methodology}

The methodology is illustrated in Fig. 1. At the first stage (corresponding with the upper box of Fig. 1), the FE model, able to predict both cutting forces and residual stresses, was developed and validated with experimental data. The Jonhson-Cook [12] constitutive law (see Eq. 1) was used in this work due to its ability to simulate mechanical processes involving high strain and strain rate and thermal softening. This constitutive law is commonly used in simulations of cutting processes [13].

$$
\sigma_{Y}=\left(A+B\left(\varepsilon^{\mathrm{pl}}\right)^{n}\right) \cdot\left(1+C \ln \left(\frac{\dot{\varepsilon}^{\mathrm{pl}}}{\dot{\varepsilon}}\right)\right) \cdot\left(1-\left(\frac{\theta-\theta_{\mathrm{ref}}}{\theta_{\text {melt }}-\theta_{\text {ref }}}\right)^{m}\right)
$$

being $\sigma_{Y}$, equivalent stress; $A$, yield limit; $B$, strain hardening coefficient; $n$, strain hardening exponent; $C$, strain rate hardening coefficient; $m$, thermal softening coefficient; $\varepsilon^{\mathrm{pl}}$, equivalent plastic strain; $\dot{\varepsilon}^{\mathrm{pl}}$, equivalent plastic strain rate; $\dot{\varepsilon}_{0}$, reference strain rate; $\theta$, temperature; $\theta_{\text {ref }}$, reference temperature; and $\theta_{\text {melt }}$, melting temperature.

Cutting tests of AISI 316L were used for the validation of the FE model. This alloy is similar (mechanical behaviour and physical properties) to those materials that will be studied in the last section of the paper. The experimentally validated FE model was used to generate a large dataset for the second stage. This data set was obtained by varying the parameters of constitutive equation, $A, B$ and $n$; and the friction coefficient at the interface, $\mu$. The output variables were the cutting and thrust force, $F_{c}$, and $F_{t}$, and the level of tensile residual stresses in machined surface in direction $1, \sigma_{11}$, and in direction $3, \sigma_{33}$. (see Fig. 2). These directions correspond, respectively, with circumferential and axial directions of the orthogonal cutting tests performed to validate the FE model. Cutting forces are significant parameters related with the mechanical behaviour of the workpiece material, and they are relatively easy to measure during machining tests. Residual stresses in machined surface, which should be measured in a post mortem analysis of the machined specimen, are related with both mechanical and thermal phenomena during cutting. The sensibility of residual stress to variations of constitutive parameters $A, B$ and $n$, have been analyzed recently in scientific literature [14]. Also the terms of the constitutive equation related with strain rate sensitivity and thermal softening ( $C$ and $m$, respectively) could influence the residual stresses and the cutting forces. However, the prediction of these parameters would involve a large number of simulations. Thus, despite of the interest of a full approach involving all terms of constitutive equation (strain hardening, strain rate hardening and thermal softening) this work is restricted to the three coefficients $A, B, n$.

At the second stage (corresponding with intermediate box of Fig. 1, see also Fig. 3) RBF and LPR models are estimated by using the dataset from FE model. The input and output variables of these models are the same used in FE model. That is, the input variables are the parameters $A, B$ and $\mu$, and the output variables were $F_{c}$, $F_{t}, \sigma_{11}$ and $\sigma_{33}$.

Finally, at the third stage (lowest box in Fig. 1, see also Fig. 4) the inverse identification is performed. During this stage, an optimization problem was solved. Now the input variables are a set of specific values of $F_{c}, F_{t}, \sigma_{11}$, $\sigma_{33}$ and $\mu$. The objective is to find the values of $A, B$ and $n$ according those input values. The estimated RBFN and LPR models of the second stage are used to related input and output variables. At the iteration $i$ of the optimization problem, a set of random values $A_{(i)}, B_{(i)}$ and $n_{(i)}$ along 


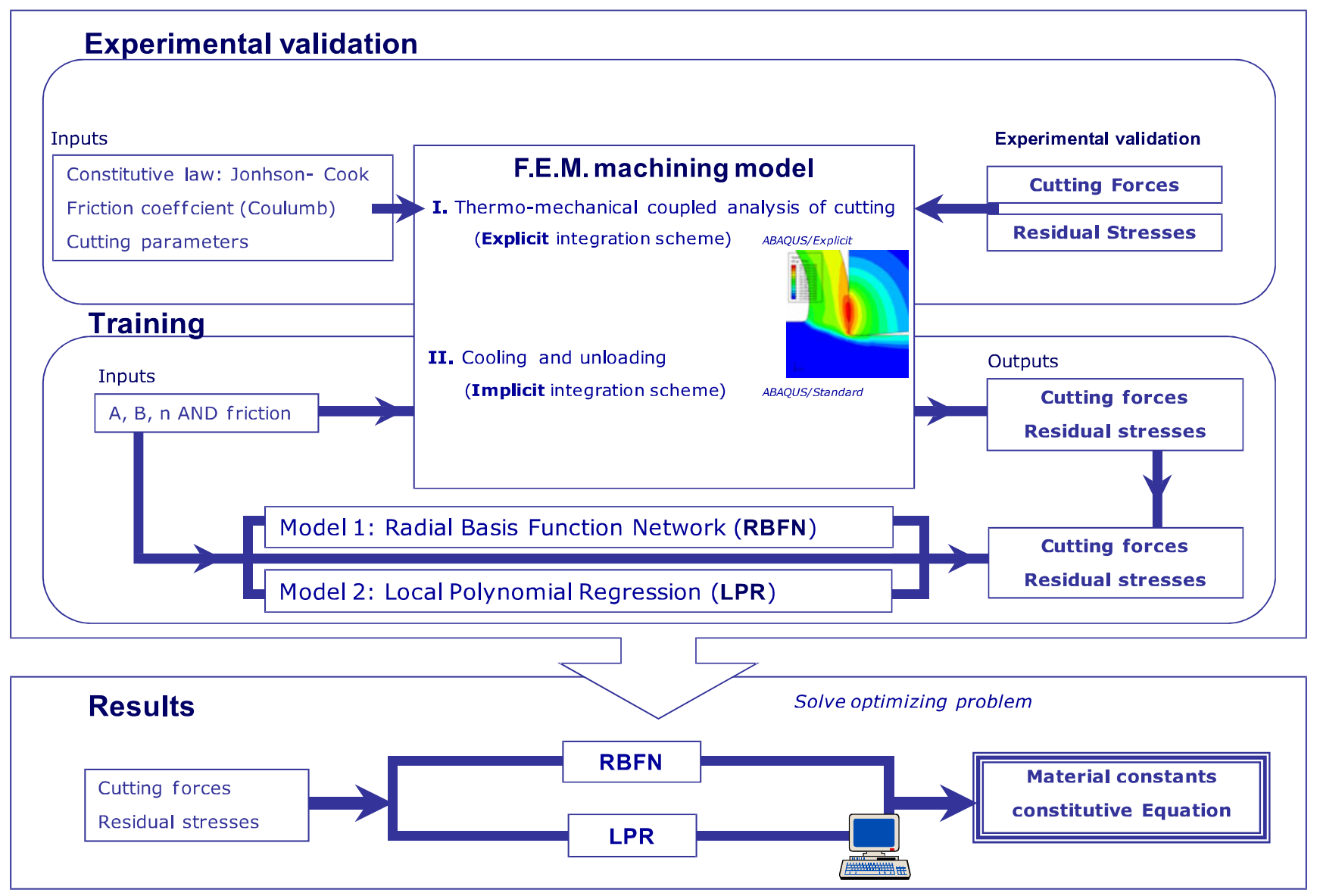

Fig. 1 Scheme of the hybrid methodology using nonparametric approach and FE modelling

with the value $\mu$ are the input variables of RBFN or LPR models. For these values, the corresponding $\widehat{F}_{c(i)}, \widehat{F}_{t(i)}$, $\widehat{\sigma}_{11(i)}$ and $\widehat{\sigma}_{33(i)}$ are estimated. The values $A_{(i)}, B_{(i)}$ and $n_{(i)}$ that minimize the difference between $\widehat{F}_{c(i)}, \widehat{F}_{t(i)}, \widehat{\sigma}_{11(i)}$ and $\widehat{\sigma}_{33(i)}$ and $F_{c}, F_{t}, \sigma_{11}, \sigma_{33}$, are the predicted constitutive material parameters.
Concerning the limitations of the method proposed, it should be noted that the RBFN and LPR models could be used to predict parameters $A, B$ and $n$, corresponding to materials showing thermal softening and strain rate hardening behaviour similar to those used during the training process of the models. Further application of the
Fig. 2 Boundary conditions and type of contour used in the model. Regions 1, 2, 3 combined sliding and Lagrangian/ Eulerian boundaries; region 4 was Eulerian

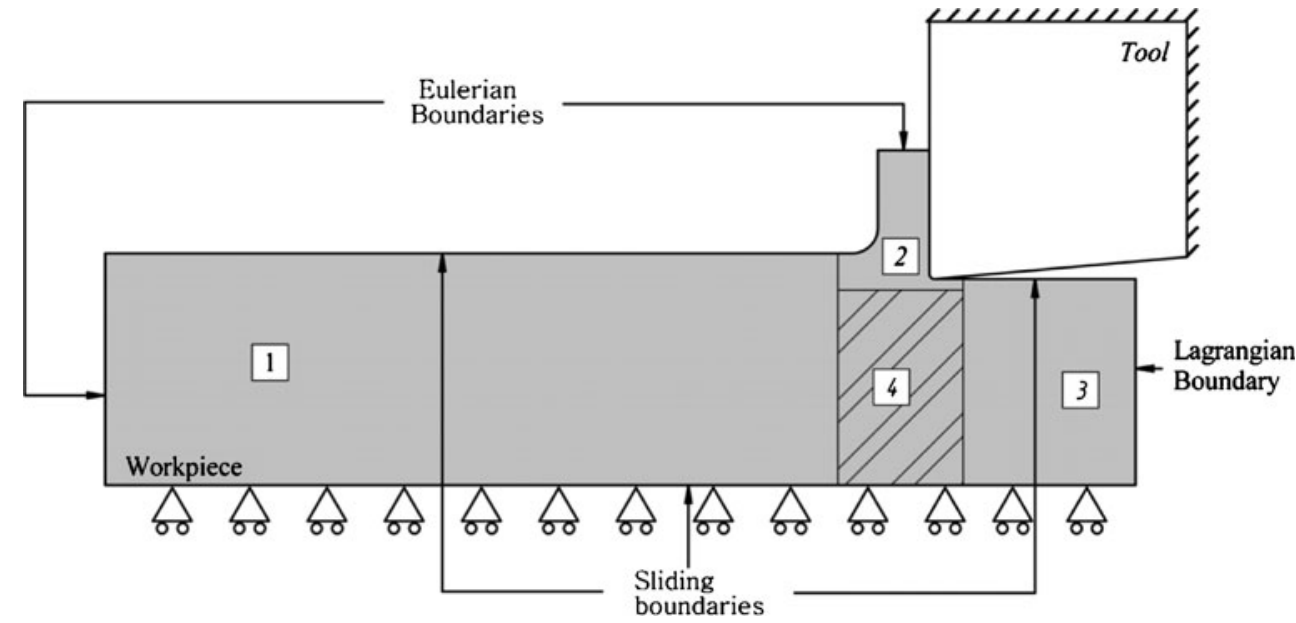




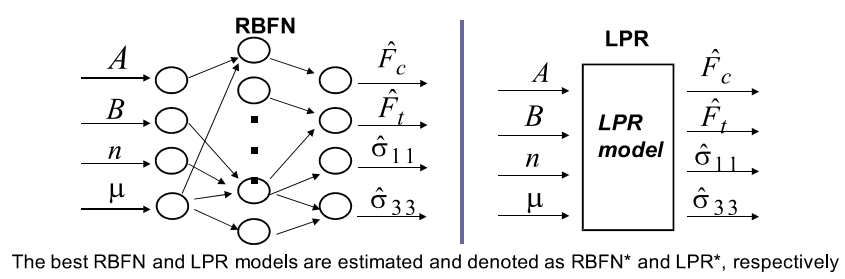

Fig. 3 Stage 2 of the methodology: RBFN and LPR model estimation

proposed methodology to predict also visco-plasticity and thermal softening terms of the constitutive equation, should involve the generation of wider amount of data from numerical models and, the training of the models varying the corresponding parameters in Eq. 1 .

\section{Description of FE model and experimental validation}

\subsection{Finite elements model}

A plane strain Arbitrary Lagrangian Eulerian (ALE) model was developed using the commercial finite element code ABAQUS/Explicit [15]. A thermo-mechanical coupled analysis was performed using the CPE4RT element type, being plane strain, quadrilateral, linearly interpolated, and thermally coupled for ALE formulation [16].

One of the most important requirements for the model was avoiding mesh distortion for all sets of parameters $A$, $B, n$ simulated. In addition, the residual stress distribution in the machined surface had to be stabilized and steady state conditions reached. The ALE model uses sliding, Lagrangian and Eulerian contours allowing the material to flow across an internal Eulerian zone surrounding the tool tip (see Fig. 2). This approach avoided extreme mesh distortion, allowing the simulation of a long machined surface (larger than $10 \mathrm{~mm}, 100$ times the value of the uncut thickness of the chip equal to $0.1 \mathrm{~mm}$ ). The details about the boundary conditions of the model (Eulerian at the entrance of the workpiece and at the exit of the chip; and Lagrangian at the end of the workpiece) can be found in
Miguélez et al. [17] with a brief description of the main advantages obtained with the implementation of these boundary conditions.

Feed rate was $0.1 \mathrm{~mm} / \mathrm{rev}$, and the cutting edge radius was $20 \mu \mathrm{m}$. Cutting speed was fixed equal to $120 \mathrm{~m} / \mathrm{min}$ for all calculations performed in the paper. Although cutting forces and residual stresses are dependent on this parameter [18], the paper is not focused on this phenomenon.

A value of the Quinney-Taylor coefficient equal to 0.9 was assumed. An initial temperature of $293 \mathrm{~K}$ was imposed. Conduction and convection to air (only in freshly machined surface) were taken into account, while radiation was neglected. The coefficient of heat convection was $20 \mathrm{~W} / \mathrm{m}^{2} \mathrm{~K}$ and sink temperature was $293 \mathrm{~K}$. Thermal flux was allowed in the attached contour of the tool [17].

Concerning the contact properties at the tool-chip interface, a constant friction coefficient along the tool/ workpiece contact length is assumed. Although it is a simple approach it has been widely used in metal cutting simulation [19].

The friction coefficient was stated as an input parameter for the non-parametric models, thus the value was varied in the range $0.3-0.8$, in order to generate numerical results (cutting forces and residual stresses) dependent on friction. The heat partition between tool and workpiece was assumed to be $50-50 \%$.

The analysis was carried out in two steps: cutting, using an explicit integration scheme and cooling and unloading, using an implicit integration scheme proposed in [20]. The cutting forces are obtained from the first step. The residual stress distribution (in directions 1 and 3, see Fig. 2), was obtained from de second step, in a section of the workpiece corresponding to stationary conditions during cutting.

As was explained before, the workpiece material was modelled using the Johnson-Cook constitutive model. The starting point for initial simulations and experimental validation of the model was a constitutive law obtained from literature for the workpiece material, AISI 316L [3], presented in Table 1.

Fig. 4 Stage 3 of the methodol-

Optimization problem, at iteration $i$

ogy: prediction of $A, B$ and

$n$ using RBFN and LPR

models

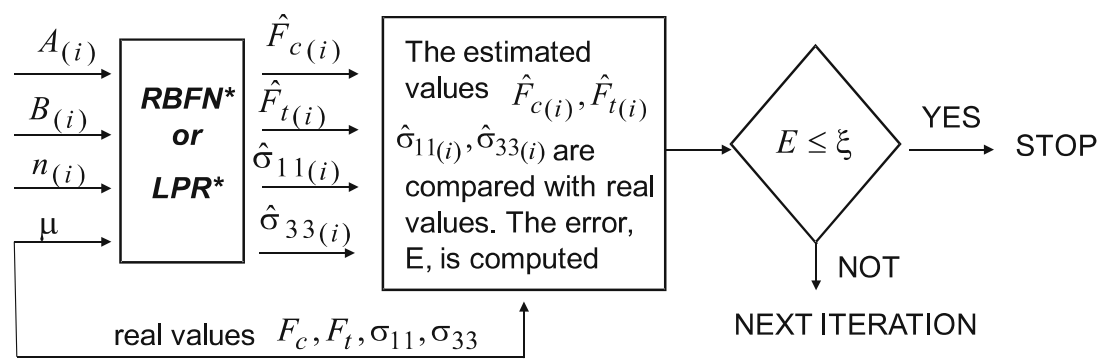


Table 1 Constitutive parameters for AISI 316L [3]

\begin{tabular}{llllll}
\hline$A(\mathrm{MPa})$ & $B(\mathrm{MPa})$ & $n$ & $C$ & $m$ & $\dot{\varepsilon}_{0\left(s^{-1}\right)}$ \\
\hline 514 & 514 & 0.508 & 0.042 & 0.533 & $10^{-3}$ \\
\hline
\end{tabular}

Physical properties of cutting material (carbide) and workpiece material were obtained from scientific literature (references $[14,21]$ ) and are presented in Tables 2 and 3.

\subsection{Experimental validation}

The model was validated with experimental data obtained from orthogonal cutting tests (AISI316L) and subsequent measurement of residual stresses in the machined surface. The value of the friction coefficient equal to 0.4 reproduced accurately the resultant residual stresses observed in experimental tests performed in the laboratory.

Orthogonal dry cutting tests with air cooling were carried out in a lathe (PINACHO model Smart-Turn 6/ $165)$ with the following parameters: cutting speed $120 \mathrm{~m} /$ $\mathrm{min}$, feed rate $0.1 \mathrm{~mm} / \mathrm{rev}$, depth of cut equal to $2 \mathrm{~mm}$. The workpiece material was a tube of AISI $316 \mathrm{~L}$ steel with a wall thickness equal to $2 \mathrm{~mm}$.

The tool geometry was generated by electro-discharge machining in a hard metal preform. Cutting edge radius was $20 \mu \mathrm{m}$. The rake angle and the clearance angle were respectively $0^{\circ}$ and $5^{\circ}$, obtained by positioning the insert in the tool holder. The support of the tool was instrumented with strain gauges allowing measurement of the cutting force during orthogonal cutting of the tube (see Fig. 5). The cutting edge of the insert was located coaxially with the axis of the support, avoiding the effect of torque during cutting. Cutting and trust forces predicted with numerical model matched reasonably the values measured in the cutting tests in the lathe. Errors below 15\% were obtained for cutting force prediction. However, the model does not predict accurately thrust force. As is well known, the inaccuracy in predicting thrust force is a common problem of FE cutting models.

The distribution of residual stress in circumferential direction was measured in the Technological Centre IDEKO (see http://www.ideko.es). The in depth distribution of residual stresses, both in circumferential and axial directions, were approximately predicted with the model

Table 2 Tool properties [21]

\begin{tabular}{ll}
\hline Properties & Carbide tool \\
\hline Density $\left(\mathrm{kg} / \mathrm{m}^{3}\right)$ & 14,900 \\
Specific heat $\left(\mathrm{J} / \mathrm{kg}^{\circ} \mathrm{C}\right)$ & 138 \\
Thermal conductivity $\left(\mathrm{W} / \mathrm{m}^{\circ} \mathrm{C}\right)$ & 79 \\
\hline
\end{tabular}

Table 3 Material properties [14]

\begin{tabular}{ll}
\hline Properties & AISI $316 \mathrm{~L}$ \\
\hline Young modulus $(\mathrm{GPa})$ & 202 \\
Poisson coefficient & 0.3 \\
Density $\left(\mathrm{kg} / \mathrm{m}^{3}\right)$ & 7,800 \\
Specific heat $\left(\mathrm{J} / \mathrm{kg}^{\circ} \mathrm{C}\right)$ & 542 \\
Thermal expansion coefficient & $1.99 \mathrm{E}-5$ \\
Thermal conductivity $\left(\mathrm{W} / \mathrm{m}^{\circ} \mathrm{C}\right)$ & 20 \\
\hline
\end{tabular}

(see Fig. 6). Experimental tensile value in the machined surface is larger than predicted value (error around $20 \%$ ). This behaviour could be related with the effect of the previous cut that appears in the turning tests. In the experimental tests used to validate simulations, the main cutting edge cuts the machined surface generated in the previous revolution of the lathe. In the case of difficult to cut material like AISI $316 \mathrm{~L}$, this zone is strongly affected by the previous cut [22] increasing the induced residual stresses in the workpiece. This increment could explain the differences observed between predicted and measured residual stresses.

\section{Theoretical concepts of RBFN and LPR}

The RBFN and LPR are nonparametric techniques to estimate the conditional expectation of a response variable, $Y$, given a set of $p$ predictor variables $X=\left(X_{1}, X_{2}, \ldots, X_{p}\right)^{\prime}$.

RBFN is a feed-forward network with three layers: input, hidden and output layer [23]. The input layer is composed of the input variables, $x$. The hidden layer of $J$ units transforms the data from the input space by applying a kernel function, $K(\bullet)$. The output $v_{j}$ of the $j$ th hidden unit is obtained as:

$v_{j}=K\left(\frac{\left\|\mathbf{X}-\mathbf{C}_{j}\right\|}{T_{j}}\right)$

where $\boldsymbol{C}_{j}$ is the vector of centres, $T_{j}$ controls the width of the kernel function and $\|\bullet\|$ denotes the Euclidean distance. Thus, $v_{j}$ is near to 1 when the input vector is near to the centre, and as the input vector is moved away from the centre, $v_{j}$ decreases. Finally, the output layer applies a linear combination of all hidden layer outputs. That is, the $k$ th output unit has the output $\widehat{Y}_{k}$ obtained as:

$\widehat{Y}_{k}=w_{0 k}+\sum_{j=1}^{J} w_{j k} v_{j}$

where $w_{0 k}$ and $w_{j k}$ are weights associated with the output unit $k$. The degree of accuracy of the RBFN can then be controlled by the shape of the kernel function, the number 
Fig. 5 Orthogonal cutting device used for testing: a $\mathrm{CNC}$ lathe $\mathbf{b}$ cutting insert attached to the instrumented tool holder a

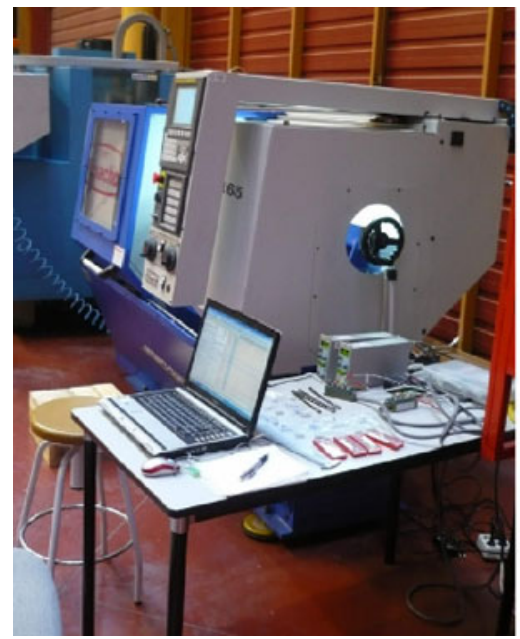

b

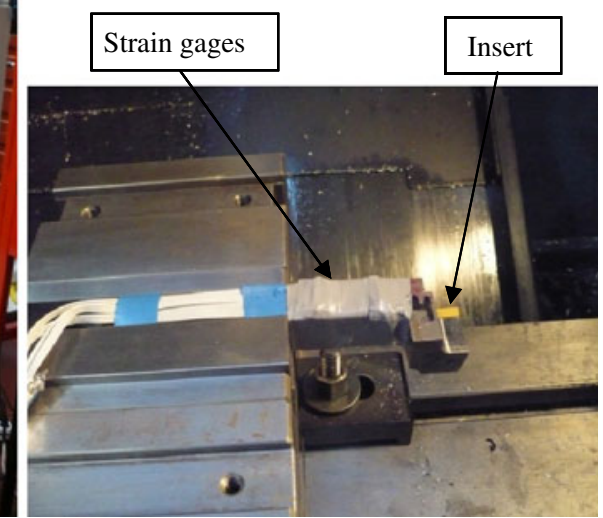

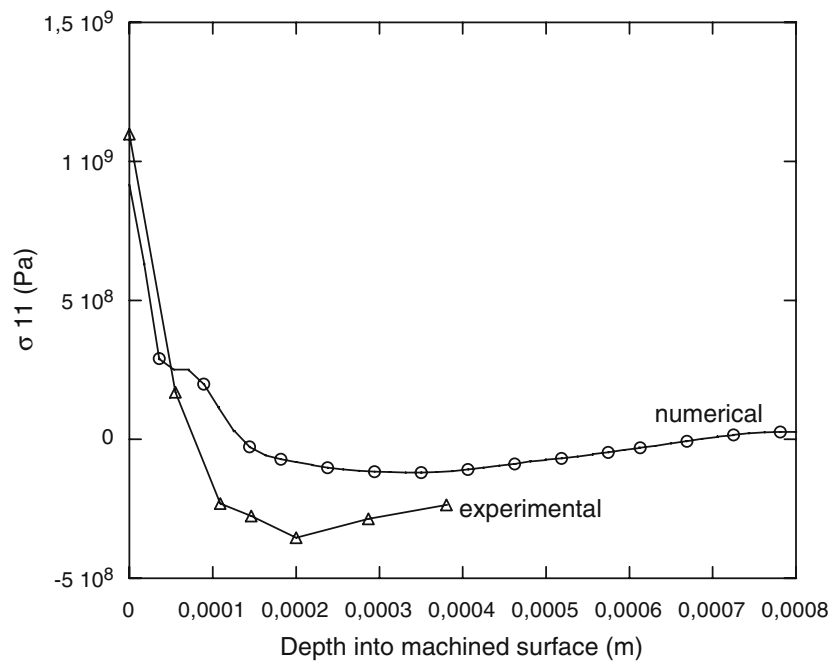

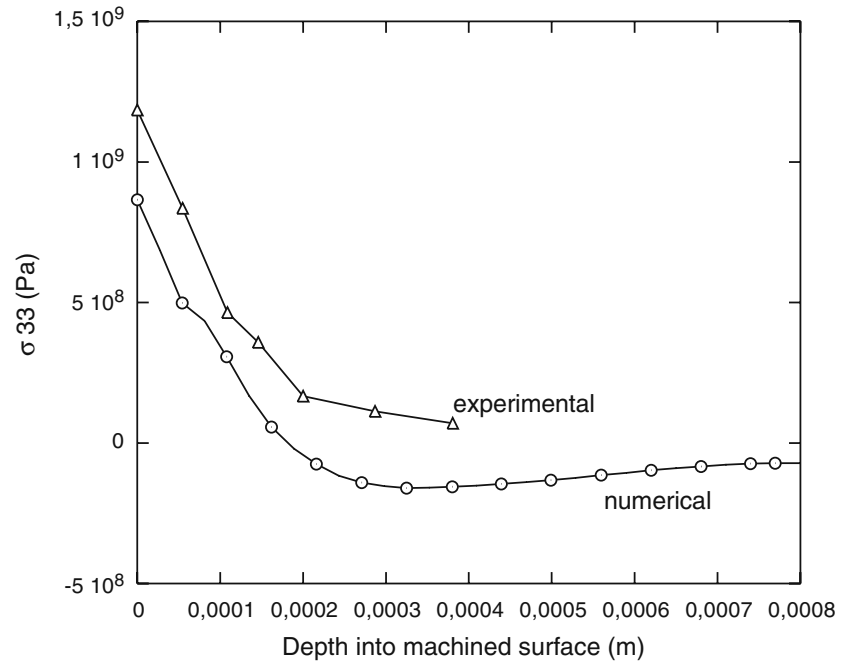

Fig. 6 Experimental and numerical residual stresses in circumferential and axial directions (corresponding with directions 1 and 3 in the numerical model, respectively) of hidden units, their centres and their widths, and the output layer weights. A training data set should be used to carry out the model parameters selection (learning stage).

LPR is a statistical approach to fitting curves to data by smoothing [7]. The fit at $X=x$ is the value of a polynomial function fitted only to those observations in a neighbourhood of $x$. LPR uses weighted least squares regression to locally fit a $d$ th degree polynomial to data. For example, for univariate predictor variable, $X$, and polynomials of degree $d=2$, the LPR model minimizes:

$\sum_{i=1}^{n} W_{i}\left(y_{i}-\beta_{0}-\beta_{1}\left(x_{i}-x\right)-\beta_{2}\left(x_{i}-x\right)^{2}\right)^{2}$

where $\left\{x_{i}, y_{i}\right\}, i=1,2, \ldots, n$, are the $n$ sample observations, $w_{i}$ is the weight for the $i$-th observation, and $\beta_{j}$, s are the model parameters. The predicted value with this model is, then, $\widehat{f}(x)=\widehat{y}=\widehat{\beta}$. The weights $w_{i}$ depend on the distance between $x_{i}$ and $x$ and are defined as:

$W_{i}=h^{-1} K\left(\frac{x_{i}-x}{h}\right)=K_{h}\left(u_{i}\right)$

where $K_{h}(\bullet)$ is a kernel function, similar to the one used in RBFN. In this case, the parameter $h$ is a smoothing parameter called the bandwidth, and the centre is the value $x$ at which the response variable $Y$ is to be estimated. The smoothing parameter regulates the size of the neighbourhood around $x$.

In the case of multivariate predictor variables, $X=\left(X_{1}, X_{2}, \ldots, X_{p}\right)^{\prime}$, the kernel function can be computed as the product of univariate kernels. That is, the kernel denoted as $K(\bullet)$ can be obtained as:

$K_{H}(u)=K_{h_{1}}\left(u_{1}\right) \cdot \ldots \cdot K_{h_{p}}\left(u_{p}\right)$

where $K_{h}\left(u_{j}\right)$ is the univariate kernel for the variable $X_{j}$. The bandwidths, $h_{j}$, can be the same or not for all univariate kernel functions. 
Table 4 Intervals of input parameters for numerical simulations and corresponding intervals of output parameters used for training non parametric models

\begin{tabular}{lccc}
\hline Input parameters & \multicolumn{3}{c}{ Output parameters } \\
\hline$A(\mathrm{MPa})$ & $300-600$ & $F_{c}(\mathrm{~N} / \mathrm{mm})$ & $156-470$ \\
$B(\mathrm{MPa})$ & $500-900$ & $F_{t}(\mathrm{~N} / \mathrm{mm})$ & $76-386$ \\
$n$ & $0.3-0.6$ & $\sigma_{11}(\mathrm{MPa})$ & $162-1,540$ \\
$\mu$ & $0.3-0.8$ & $\sigma_{33}(\mathrm{MPa})$ & $272-1,420$ \\
\hline
\end{tabular}

The accuracy of the LPR model is, then, controlled by the shape of the kernel functions, the bandwidth $h_{j}$ and the polynomial degree $d$. As in RBFN models, a training data set should be used in order to select these model parameters.

\section{Modelling results and discussion}

\subsection{First stage: finite element results}

Numerical simulations were performed with different sets of Johnson-Cook equation parameters and values of friction coefficient. Total number of sets used in numerical simulations was 320 , large enough to train both non- parametric models (RBFN and LPR). The values of the terms of the constitutive equation were ranged from the initial values (given in [3]); Table 4 shows the intervals of $A, B, n$ and friction $\mu$, implemented in the numerical work. The intervals were selected covering representative values characteristics of different type of steel behaviour found in scientific literature.

Residual stresses results from the interaction of different coupled phenomena. Temperature increase implies both thermal softening and enhanced thermal expansion contribution, having opposite effects in resultant residual stress [11]. During cutting simulation, significant influence of input parameters in chip morphology and stress and temperature fields was observed. Some examples of the temperature and $\sigma_{11}$ fields during cutting are shown in Figs. 7 and 8. In the simulations presented in Fig. 7, the same value of parameters $A=514$ and $B=800 \mathrm{MPa}$ were considered, while $n$ was equal to $0.4,0.5$ and 0.6 . Figure 8 shows the influence of $B$, being equal to 514 and $800 \mathrm{MPa}$, respectively, while the other parameters were $A=514 \mathrm{MPa}$ and $n=0.5$. The increment of $A$, being the elastic limit, led to decreased values of tensile residual stresses. The increment of $\mathrm{B}$ and $\mathrm{n}$, representing increased hardening originated larger values of residual stresses. These trends observed in this work are in agreement with those presented in [20].
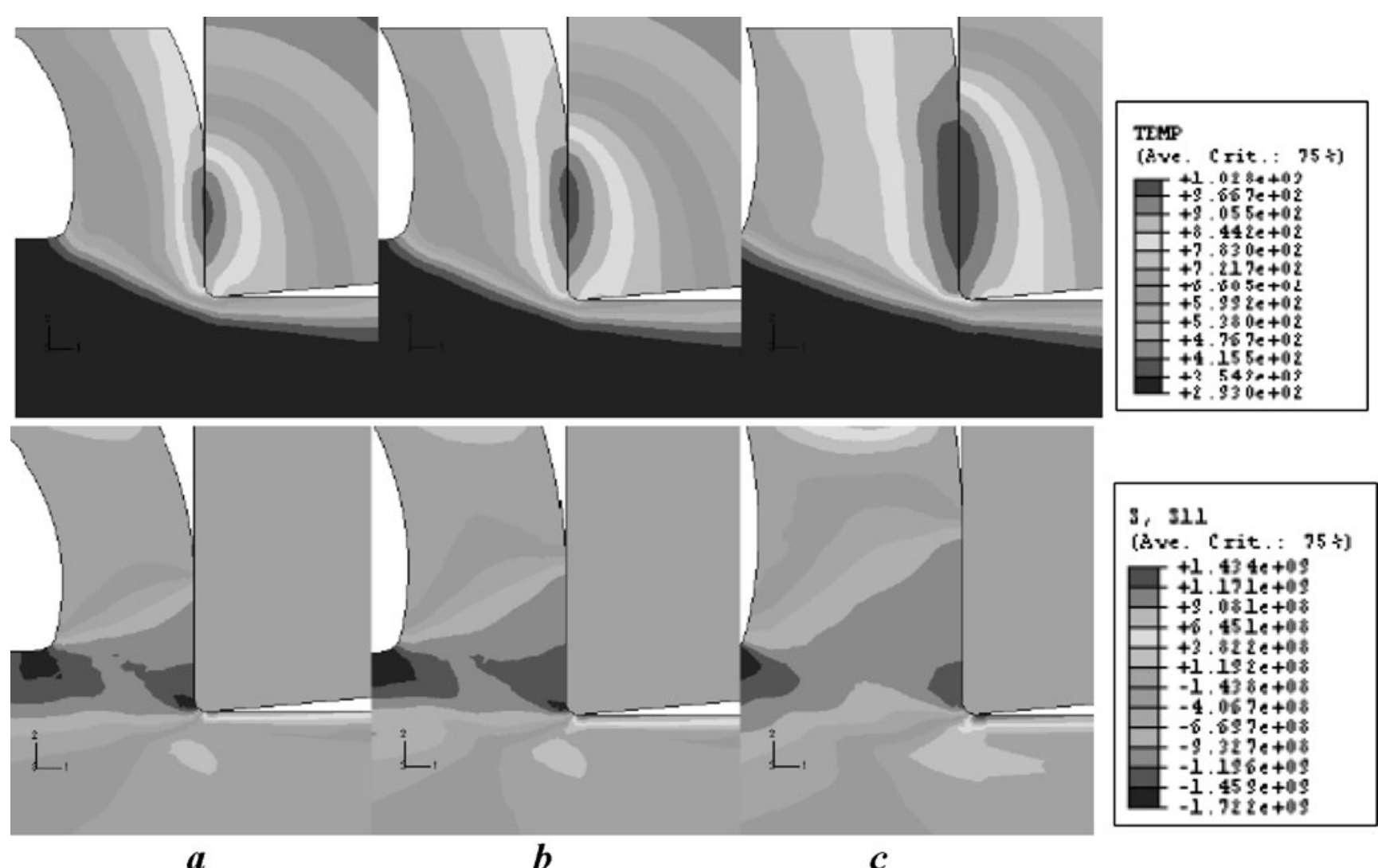

Fig. 7 Temperature (K) and stress field $\sigma_{11}$ (Pa, direction 1) obtained for $A=514 B=800$ a $n=0.4$ b $n=0.5$, c $n=0.6$ 


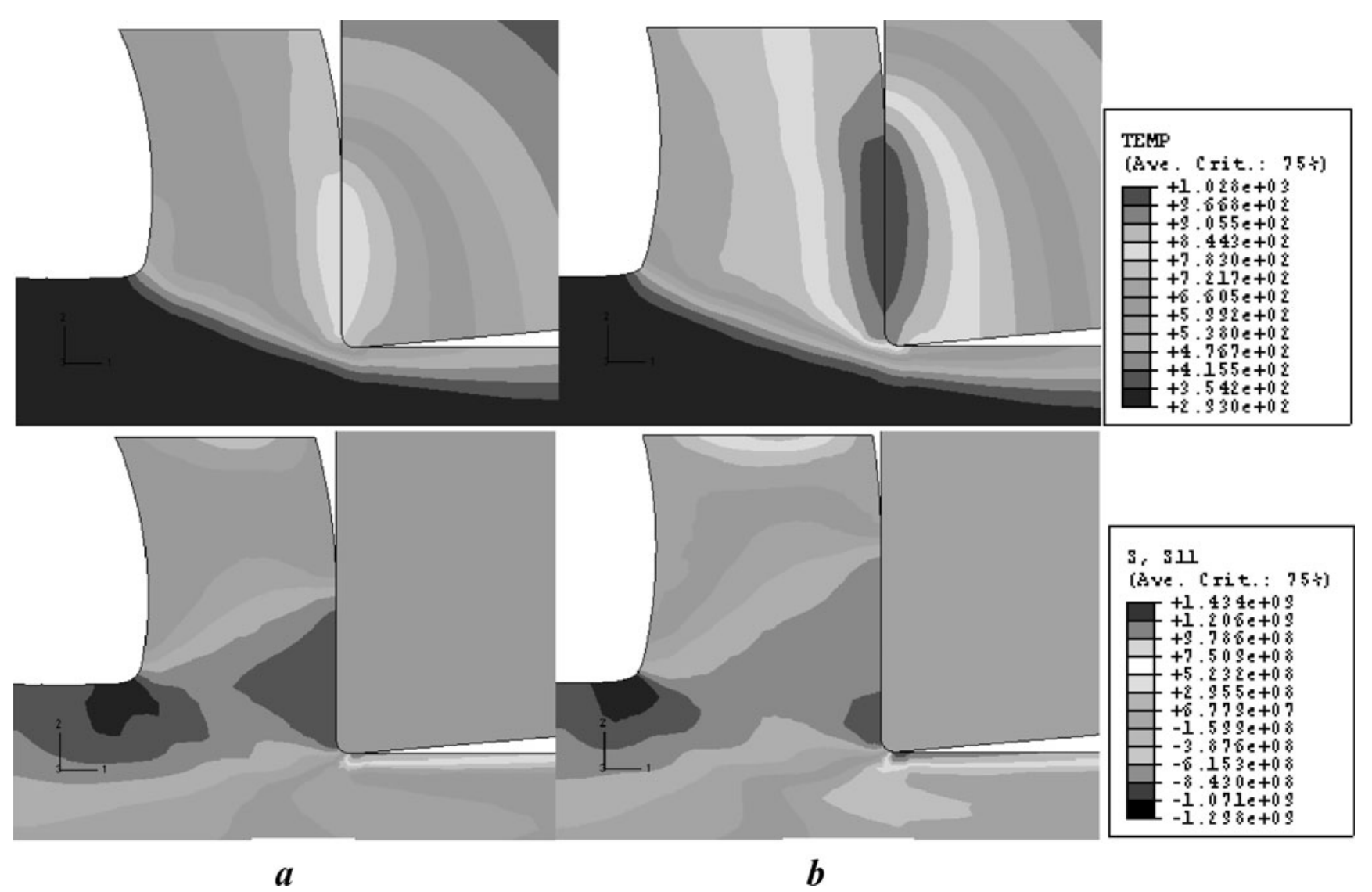

Fig. 8 Temperature (K) field and stress field $\sigma_{11}$ (Pa, direction 1), $A=514, n=0.5$, a $B=514 \mathbf{b} B=800$

\subsection{Second stage: RBFN and LPR models estimation}

In order to find the best RBFN model, as well as, the best LPR model, the dataset of $N=320$ cases generated from FEM is used. The selection of the best models is based on Monte Carlo cross-validation (MCCV) technique [24]. Figure 3 illustrates this stage.

The MCCV is a method for estimating the prediction error based on resampling. In MCCV, the dataset, of $N$ observations, is randomly split into two parts. The first part, of $N-N_{v}$ observations, is denoted as training set and used to fitting the model. The second part, of $N_{v}$ observations, is denoted as validation set and used to measure how well the model fits this new data, that is, to compute the prediction error. This procedure is repeated a large number of times, $M$. Then, the best model is the one with the smallest average prediction error, computed based on the $M$ different ways of data splitting.

Here, two metrics, the mean square error (MSE), and the mean absolute relative error (MARE), were used to evaluate the alternative models. Then, the global errors of the model, $\mathrm{MSE}_{G}$ and $\mathrm{MARE}_{G}$, are computed as:

$$
\begin{aligned}
& \operatorname{MSE}_{G}=\left(s N_{v} M\right)^{-1} \sum_{i=1}^{M} \sum_{j=1}^{s} \sum_{k=1}^{N_{v}}\left(y_{i j k}-\widehat{y}_{i j k}\right)^{2} \\
& \operatorname{MARE}_{G}=\left(s N_{v} M\right)^{-1} \sum_{i=1}^{M} \sum_{j=1}^{s} \sum_{k=1}^{N_{v}} \frac{\left|y_{i j k}-\widehat{y}_{i j k}\right|}{y_{i j k}}
\end{aligned}
$$

Table 5 MSE and MARE of best RBFN and LPR models

\begin{tabular}{lllllll}
\hline & & $F_{c}$ & $F_{t}$ & $\sigma_{11}$ & $\sigma_{33}$ & MSE $_{G}$ or $\mathrm{MARE}_{G}$ \\
\hline RBFN & MSE & 0.00041 & 0.00051 & 0.00171 & 0.00164 & 0.00107 \\
& MARE & 0.02036 & 0.02371 & 0.06640 & 0.05216 & 0.04066 \\
\multirow{2}{*}{ LPR } & MSE & 0.00035 & 0.00048 & 0.00158 & 0.00152 & 0.00098 \\
& MARE & 0.01875 & 0.02296 & 0.06241 & 0.04963 & 0.03844 \\
\hline
\end{tabular}


Table 6 APE (\%) of the constitutive parameters using RBFN model

\begin{tabular}{|c|c|c|c|c|c|c|c|c|}
\hline$A$ & $\widehat{A}$ & APE (\%) & $B$ & $\widehat{B}$ & APE (\%) & $n$ & $\widehat{n}$ & APE (\%) \\
\hline 363 & 302.27 & 16.73 & 742 & 775.81 & 4.56 & 0.46 & 0.40 & 12.78 \\
\hline 385 & 387.68 & 0.70 & 533 & 500.00 & 6.19 & 0.38 & 0.46 & 20.82 \\
\hline 457 & 491.95 & 7.65 & 624 & 764.34 & 22.49 & 0.44 & 0.39 & 10.25 \\
\hline 464 & 549.13 & 18.35 & 818 & 847.45 & 3.60 & 0.57 & 0.51 & 9.84 \\
\hline 471 & 366.81 & 22.12 & 752 & 851.26 & 13.20 & 0.41 & 0.35 & 14.17 \\
\hline 532 & 509.24 & 4.28 & 596 & 672.92 & 12.91 & 0.52 & 0.51 & 2.38 \\
\hline 565 & 563.23 & 0.31 & 810 & 852.39 & 5.23 & 0.33 & 0.31 & 5.48 \\
\hline 567 & 587.25 & 3.57 & 876 & 899.90 & 2.73 & 0.34 & 0.32 & 6.18 \\
\hline 592 & 542.53 & 8.36 & 846 & 713.84 & 15.62 & 0.54 & 0.59 & 8.94 \\
\hline 641 & 614.07 & 4.20 & 641 & 889.94 & 38.84 & 0.55 & 0.44 & 19.24 \\
\hline
\end{tabular}

where, $s$ is the number of output variables, $y$ is the real value, and $\hat{y}$ the estimated value.

The results showed in this section are based on a validation set of $N_{v}=25$ observations, and a value of $M=$ 5,000 iterations. The number of output variables is $s=4$. Because variables span over different ranges, all variables are normalized between 0 and 1 . Since the selection of kernel functions is not critical to the performance of the nonparametric fit [7], the kernel in all models (RBFN or LPR) is the Gaussian function.

Therefore, for RBFN models, the parameters that should be determined were $T_{j}, C_{j}, w_{j k}$, in Eqs. (2) and (3), and the number of hidden neurons, $J$. For LPR models, the parameters were the bandwidths $h_{j}$, in Eq. (6), and the polynomial degree $d$.

In the case of LPR models, since variables have been normalized between $[0,1]$, all bandwidths are set the same, that is, $h_{j}=h, j=1,2,3,4$.

For RBFN models, the orthogonal least square (OLS) is used as learning procedure [25]. The OLS uses the same value $T_{j}$ (that is, $T_{j}=T$ ) for all hidden neurons. For a given $T$, the algorithm optimized the number of hidden neurons, $J$, their centres $\boldsymbol{C}_{j}$, and the output layer weights, $w_{j k}$. Hence, in order to select the best RBFN model, only the parameter $h$ is controlled.

The Matlab is used for this analysis. The parameters of the best estimated models were the following:

- RBFN: $T=2.27$ and $J=120$ hidden neurons. The location of the centres $\boldsymbol{C}_{j}$, and the output weights, $w_{j k}$, are not listed for simplicity.

- LPR: $h=0.13$ and $d=3$.

Table 2 shows the MSE and the MARE for these models. We can conclude that both models have similar performance with LPR showing a better overall performance. Both metrics have very low values, showing the efficiency of the models. The MARE for variables $F_{c}$, and $F_{t}$, has a value around $2 \%$. In the case of variables $\sigma_{11}$ and $\sigma_{33}$, this error has a value around $6 \%$ and $5 \%$, respectively.

5.3 Third stage: prediction of constitutive parameters $A, B$ and $n$

In this stage, a set of $F_{c}, F_{t}, \sigma_{11}$, and $\sigma_{33}$, as well as, the parameter $\mu$ (obtained as the ratio between thrust and

Table $7 A P E(\%)$ of the constitutive parameters using LPR model

\begin{tabular}{|c|c|c|c|c|c|c|c|c|}
\hline A & $\widehat{A}$ & APE $(\%)$ & $B$ & $\widehat{B}$ & APE (\%) & $n$ & $\widehat{n}$ & APE (\%) \\
\hline 363 & 430.20 & 18.51 & 742 & 725.42 & 2.23 & 0.46 & 0.48 & 4.74 \\
\hline 385 & 355.93 & 7.55 & 533 & 500.10 & 6.17 & 0.38 & 0.45 & 18.34 \\
\hline 457 & 524.92 & 14.86 & 624 & 627.31 & 0.53 & 0.44 & 0.45 & 1.84 \\
\hline 464 & 402.08 & 13.34 & 818 & 807.46 & 1.29 & 0.57 & 0.56 & 2.30 \\
\hline 471 & 379.84 & 19.35 & 752 & 857.07 & 13.97 & 0.41 & 0.35 & 15.15 \\
\hline 532 & 552.97 & 3.94 & 596 & 556.21 & 6.68 & 0.52 & 0.57 & 9.96 \\
\hline 565 & 584.21 & 3.40 & 810 & 822.65 & 1.56 & 0.33 & 0.32 & 2.39 \\
\hline 567 & 547.99 & 3.35 & 876 & 798.91 & 8.80 & 0.34 & 0.34 & 1.41 \\
\hline 592 & 526.60 & 11.05 & 846 & 899.76 & 6.35 & 0.54 & 0.51 & 4.98 \\
\hline 641 & 646.73 & 0.89 & 641 & 629.26 & 1.83 & 0.55 & 0.58 & 5.82 \\
\hline
\end{tabular}



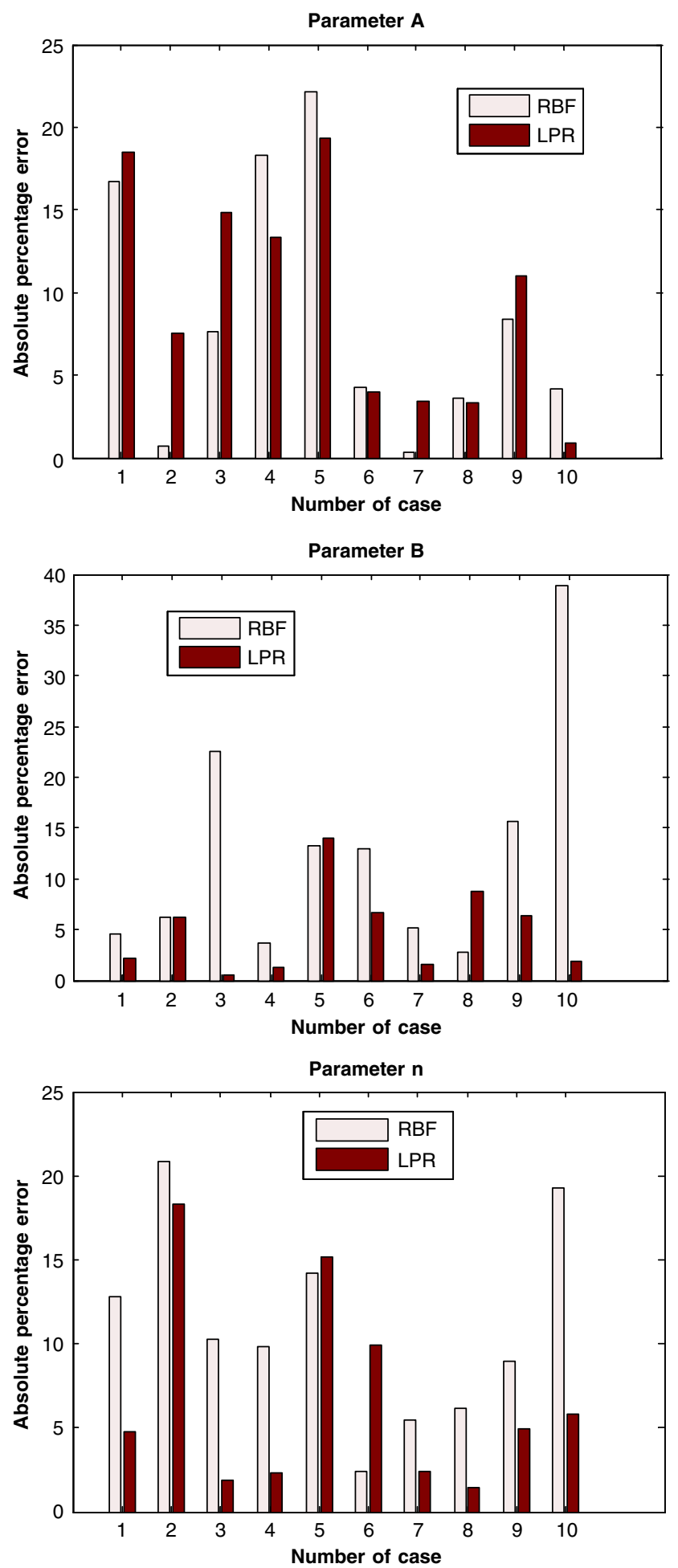

Fig. 9 Absolute percentage error obtained with both RBF and LPRN methods

cutting force) are known (Table 5). The objective is to predict the values of parameters $A, B$ and $n$. As mentioned in section 1, an optimization problem is solved. At iteration $i$, a set of random values $A_{(i)}, B_{(i)}$ and $n_{(i)}$ along with the
Table 8 MAPE (\%) of the constitutive parameters from RBFN and LPR models

\begin{tabular}{llrr}
\hline & $A$ & $B$ & \multicolumn{1}{c}{$n$} \\
\hline RBFN & 8.62 & 12.54 & 11.01 \\
LPR & 9.63 & 4.94 & 6.69 \\
\hline
\end{tabular}

value $\mu$ are used as input variables of RBFN or LPR model. Then, the values $\widehat{F}_{c(i)}, \widehat{F}_{t(i)}, \widehat{\sigma}_{11(i)}$ and $\widehat{\sigma}_{33(i)}$ are estimated. These values are used to compute the error, $E$, as:

$$
\begin{aligned}
E= & \frac{\left|F_{c}-\widehat{F}_{c(i)}\right|}{F_{c}}+\frac{\left|F_{t}-\widehat{F}_{t(i)}\right|}{F_{t}}+\frac{\left|\sigma_{11}-\widehat{\sigma}_{11(i)}\right|}{\sigma_{11}} \\
& +\frac{\left|\sigma_{33}-\widehat{\sigma}_{33(i)}\right|}{\sigma_{33}}
\end{aligned}
$$

The genetic algorithm is used to minimize the error $E$ [26]. The values $A_{(i)}, B_{(i)}$ and $n_{(i)}$ that minimize $E$ are the predicted parameters.

Here, we show the predicted parameters $\widehat{A}, \widehat{B}$ and $\widehat{n}$, for a set of ten cases. These cases are obtained from the FEM and they have not been used in stage 2 . The error used to evaluate the prediction performance is the absolute percentage error (APE):

$\operatorname{APE}(\%)=\frac{|y-\widehat{y}|}{y} \times 100$

Table 6 shows the real values of the parameters $A, B$ and $n$, the values estimated with the RBFN model, and the corresponding APE. Table 7 shows the same information for the LPR model. Figure 9 compares the errors of these tables. Table 8 summarizes the errors of Tables 6 and 7 showing the mean error (MAPE), for each parameter and each model. The MAPE is lower than $13 \%$ in the case of RBFN and lower than $10 \%$ for LPR. On the basis of these results we may conclude that LPR outperforms the RBFN model. Thus, LPR is used in the next section to predict constitutive law parameters from a set of experimental data. The procedure described in this stage is shown in Fig. 4.

\begin{tabular}{|c|c|c|c|c|c|c|}
\hline Reference & $A$ & $\widehat{A}$ & $B$ & $\widehat{B}$ & $n$ & $\widehat{n}$ \\
\hline [27] & 375 & & 552 & & 0.457 & \\
\hline [28] & 375 & & 580 & & 0.500 & \\
\hline [29] & 553.1 & & 600.8 & & 0.234 & \\
\hline [30] & 451 & & 819.5 & & 0.173 & \\
\hline Estimated values & & 427 & & 772 & & 0.21 \\
\hline
\end{tabular}

Table 9 J-C parameters for AISI 1045 


\subsection{Inverse identification from experimental data}

Since LPR model gives the better prediction, it is used for inverse estimation of constitutive law parameters of two types of steels: AISI 1045 and AISI 316L. Experimental data for these steels were obtained from the scientific literature to be used as an input for the inverse identification method.

Experimental data for stainless steel AISI 1045, obtained from comparable cutting conditions were obtained from reference [27]. The data from [27] were $F_{c}=652 \mathrm{~N} / \mathrm{mm}, F_{t}=$ $338 \mathrm{~N} / \mathrm{mm}$ and $\sigma_{11}=530 \mathrm{MPa}$. The value of $\sigma_{33}$ was considered to be approximately the same as $\sigma_{11}$, because no data was found about this parameter. Table 9 shows the estimated values for $A, B$ and $n$ obtained by using the proposed inverse identification methodology and those values of $F_{c}, F_{t}, \sigma_{11}$ and $\sigma_{33}$ and also summarizes the values of these parameters for the AISI 1045, found in the literature [28-31]. As shown in Table 9, the results provided by the proposed inverse methodology lied in the range of the available material parameters. The results were especially very close to those proposed by Özel [31].

The methodology is also applied to the data given in the work of Outeiro et al. [32] concerning the machining induced residual stresses in AISI 316L. The values used are $F_{c}=291 \mathrm{~N} / \mathrm{mm}, F_{t}=237 \mathrm{~N} / \mathrm{mm}, \sigma_{11}=900 \mathrm{MPa}$ and $\sigma_{33}=$ $600 \mathrm{MPa}$. In this case, the material constants obtained with the proposed method are shown in Table 10. These values are in the range proposed in the works [33-35] and summarized in Table 10.

In order to check the material parameters by inverse identification, the values given for both materials (AISI 1045 and AISI 316L) by the LPR model were implemented in the numerical model. Numerical output (forces and residual stresses) were compared with those presented in the references [26-32]. The error values for cutting forces obtained from numerical simulation were similar to those obtained in the experimental validation of the numerical model presented previously. The same trend was observed for residual stresses. The level of tensile residual stresses in

Table $10 \mathrm{~J}-\mathrm{C}$ parameters for AISI $316 \mathrm{~L}$

\begin{tabular}{|c|c|c|c|c|c|c|}
\hline Reference & $A$ & $\widehat{A}$ & $B$ & $\widehat{B}$ & $n$ & $\widehat{n}$ \\
\hline$[32]$ & 305 & & 1,161 & & 0.61 & \\
\hline$[32]$ & 305 & & 441 & & 0.10 & \\
\hline$[33]$ & 301 & & 1,472 & & 0.807 & \\
\hline$[34]$ & 280 & & 1,750 & & 0.8 & \\
\hline$[3]$ & 514 & & 514 & & 0.508 & \\
\hline Estimated values & & 330 & & 822 & & 0.4 \\
\hline
\end{tabular}

the machined surface was underestimated with an error close to $20 \%$; however as was explained before, this phenomenon could be related with the effect of previous passes inducing subsurface hardening in the workpiece. These results are reasonably, having into account that the errors are due not only to the estimation method. Also the inexactitude derived from the use of experimental values obtained from literature (due for instance to possible different treatments or processing routes of the workpiece material, or to uncertainties of the measurement in each experiment) influences the errors of the final result.

\section{Conclusions}

The main contribution of this paper is the exploration of the potentiality of the methodology developed, that has proved its ability to perform inverse identification of material parameters from cutting tests. RBFN and LPR models were used to identify these parameters from experimental data obtained from cutting tests. These models are useful tools in prediction of variables with complex dependence on input data. Finite element analysis was performed to train the nonparametric models. Different methodology stages were covered successfully. Firstly the numerical model was developed and validated showing reasonably accuracy when compared with experimental data. Secondly, the nonparametric models were trained with FE output and were able to predict material parameters with acceptable errors. Finally, the method was applied to experimental data (cutting forces and residual stresses) obtained from literature focused on machining of two types of stainless steel. Although LPR has not been commonly used in analysis of manufacturing processes, this method presented lower level of error. In fact the constitutive parameters obtained with the LPR model agreed reasonably with the range of values presented in literature for each material analyzed.

The proposed methodology could be easily extended to predict also strain rate hardening and thermal softening terms of the constitutive equation. Future work in the topic presented in the paper would also involve improved testing procedure with the measurement of other significant variables during cutting (mainly temperature). Furthermore, other representative parameters obtained from numerical simulation could be used, for instance the geometrical characteristics of the chip morphology, strongly dependent on constitutive law parameters. Also the numerical modelling could be improved considering 3D codes and more sophisticated contact laws or other advanced constitutive equations. The improvement of each stage involved in the presented methodology would 
lead to improvements in the final inverse identification procedure.

Acknowledgments The authors acknowledge the financial support of this work to the Ministry of Science and Education of Spain (under project DPI2008-06746).

\section{References}

1. Gonzalo O, Jauregi H, Uriarte L, López de Lacalle L (2009) Prediction of specific force coefficients from a FEM cutting model. Int J Adv Manuf Technol 43:348-356

2. Davim J, Maranhão C, Jackson M, Cabral G, Grácio J (2009) FEM analysis in high speed machining of aluminium alloy (A17075-0) using polycrystalline diamond (PCD) and cemented carbide (K10) cutting tools. Int J Adv Manuf Technol 39:1093-1100

3. Tounsi N, Vincenti J, Otho A, Elbestawi MA (2002) From the basics of orthogonal metal cutting toward the identification of the constitutive equation. Int J Mach Tools Manuf 42/2:1373-1383

4. Kuo C-FJ, Wu Y-S (2005) Application of a Taguchi-based neural network prediction design of the film coating process for polymer blends. Int J Adv Manuf Technol 27:455-461. doi:10.1007/ s00170-004-2215-3

5. Kohli A, Dixit US (2005) A neural-network-based methodology for the prediction of surface roughness in a turning process. Int $\mathrm{J}$ Adv Manuf Technol 25(1/2):118-129

6. Sanjay C, Jyothi C (2006) A study of surface roughness in drilling using mathematical analysis and neural networks. Int $\mathrm{J} \mathrm{Adv}$ Manuf Technol 29:846-852

7. Härdle W (1990) Applied nonparametric regression. Cambridge University Press, Cambridge, UK

8. Umbrello D, Ambrogio G, Filice L, Shivpuri R (2008) A hybrid finite element method-artificial neural network approach for predicting residual stresses and the optimal cutting conditions during hard turning of AISI 52100 bearing steel. Mater Design 29:873-883

9. Chamekh A, Bel Hadj Salah H, Hambli R (2009) Inverse technique identification of material parameters using finite element and neural network computation. Int $\mathrm{J}$ Adv Manuf Technol 44:173-179. doi:10.1007/s00170-008-1809-6

10. Fernández-Fdz D, Zaera R (2008) A new tool based on artificial neural networks for the design of lightweight ceramic-metal armour against high-velocity impact of solids. Int J Solid Struct 45:6369-6383

11. Miguélez MH, Zaera R, Molinari A, Cheriguene R, Rusinek A (2009) Residual stresses in orthogonal cutting of metals: the effect of thermomechanical coupling parameters and of friction. J Therm Stresses 32(3):269-289

12. Johnson GR, Cook WH (1983) A constitutive model and data for metals subjected to large strains, high strain rate and temperature. In: Proc 7th Int Symp on Ballistics. pp. 541-547.

13. Muñoz-Sánchez A, Miguélez H, Canteli JA, Cantero JL (2009) The influence of tool wear in surface integrity when machining austenitic stainless steel. In: 9th International Conference on the Mechanical and Physical Behaviour of Materials under Dynamic Loading (DYMAT 2009), Brussels, Belgium

14. Umbrello D, M'Saoubi R, Outeiro JC (2007) The influence of Johnson-Cook material constants on finite element simulation of machining of AISI 316L steel. Int J Mach Tools Manuf 47:462-470

15. Hibbit Karlson and Sorensen Inc (2003) ABAQUS User's Manual $6.4-1$
16. Adetoro OB, Wen PH (2010) Prediction of mechanistic cutting force coefficients using ALE formulation. Int $\mathrm{J}$ Adv Manuf Technol 46(1/4):79-90

17. Miguélez H, Muñoz-Sánchez A, Cantero JL, Loya JA (2009) An efficient implementation of boundary conditions in an ALE model for orthogonal cutting. J Theor Appl Mech 47(3):1-20

18. Miguélez MH, Zaera R, Molinari A, Muñoz-Sánchez A (2009) The influence of cutting speed in the residual stresses induced by HSM in AISI 316L steel, 12th CIRP Conference on Modelling of Machining Operations. San Sebastián, Spain.

19. Moufki A, Molinari A, Dudzinski D (1998) Modelling of orthogonal cutting with a temperature dependent friction law. J Mech Phys Solids 46/10:2103-38

20. Nasr MNA, Ng EG, Elbestawi MA (2008) A modified timeefficient $\mathrm{FE}$ approach for predicting machining-induced residual stresses. Finite Elem Anal Des 44:149-161

21. Jang DY, Watkins TR, Kozaczek KJ, Hubbard CR, Cavin OB (1996) Surface residual stresses in machined austenitic stainless steel. Wear 194:168-173

22. Korkut I, Kasap M, Ciftci I, Seke U (2004) Determination of optimum cutting parameters during machining of AISI 304 austenitic stainless steel. Mater Design 25:303-305

23. Haykin S (2007) Neural networks: a comprehensive foundation. Prentice Hall, Englewood Cliffs, NJ

24. Shao J (2003) Linear Model Selection by Cross-Validation. J Amer Stat Assoc 88:486-494

25. Chen S, Cowan CFN, Grant PM (1991) Orthogonal least squares learning algorithm for radial basis function networks. IIEE Trans Neur Netw 2:302-309

26. Goldberg DE (1989) Genetic Algorithms in Search, Optimization and Machine Learning. Kluwer Academic Publishers, Boston, MA

27. Zouhar J, Piska M (2008) Modelling the orthogonal machining process using cutting tools with different geometry. MM Sci J 48 51

28. Borkovec J, Petruska J (2007) Computer simulation of material separation process. In: Proc. Engineering Mechanics, Svratka, Czech Republic. p. 14.

29. Forejt M (2004) The mechanical properties of selected types of steel in the condition of higher speed deformation. Report of Research plan MSM 262100003, Brno University of Technology, Institute of Manufacturing Technology, Czech Republic

30. Jaspers SPFC, Dautzenberg JH (2002) Material behaviour in conditions similar to metal cutting: flow stress in the primary shear zone. J Mater Process Technol 122:322-330

31. Özel T, Zeren E (2005) Finite element modeling of stresses induced by high speed machining with round edge cutting tools. ASME International Mechanical Engineering Congress \& Exposition, Orlando

32. Outeiro JC, Umbrello D, M'Saoubi R (2006) Experimental and numerical modelling of the residual stresses induced in orthogonal cutting of AISI $316 \mathrm{~L}$ steel. Int J Mach Tool Manufact 46 (14):1786-1794

33. Chandrasekaran H, M'Saoubi R, Chazal H (2005) Modelling of material flow stress in chip formation process from orthogonal milling and split Hopkinson bar tests. Mach Sci Technol 9:131145

34. M'Saoubi R (1998) Aspects Thermiques et Microstructuraux de la coupe. Application a la coupe orthogonale des aciers austenitiques. Ph.D. Thesis, ENSAM-Paris

35. Changeux B, Touratier M, Lebrun JL, Thomas T, Clisson J (2001) High-speed shear tests for the identification of the Johnson-Cook law. Fourth International ESAFORM Conference. pp. $603-606$ 